\title{
Risiko von Medikamenteninteraktionen - einfach und ökonomisch erfassen
}

\author{
W. Byland*, R. Oberhänsli
}

\begin{abstract}
Medizin kann für die Patienten gefährlich sein. Interaktionen zwischen verschiedenen Medikamenten sind eine der möglichen Gefahrenquellen für die Patienten. Das Risiko steigt mit der Anzahl der verabreichten Medikamente. Eine routinemässige Überprüfung der Medikamentenliste mittels einer OnlineDatenbank ist wenig aufwendig und ergibt eine gute Information über mögliche Interaktionsrisiken. Daraus können die klinischen Konsequenzen gezogen werden, häufig sind besondere Kontrollen oder besondere Aufmerksamkeit angezeigt, selten müssen Medikamente abgesetzt oder gewechselt werden. Dies stellt einen einfach zu realisierenden Beitrag zur Verbesserung der Patientensicherheit dar.
\end{abstract}

\footnotetext{
* W. Byland / die Klinik Schützen ist, wie alle Ärztekunden, Klein-
} aktionär bei der Apotheke zur Rose.

Korrespondenz:

Dr. med. Walter Byland

Klinik Schützen

Bahnhofstrasse 19

CH-4310 Rheinfelden

E-Mail:

walter.byland@schuetzen-ag.ch

\section{Einleitung}

Ob Hippokrates oder Galen oder ein anderer es zuerst sagte, «Primum non nocere» ist als Maxime bekannt, Ärzte und Ärztinnen sollen beim Behandeln zuallererst einmal nicht schaden und danach, so es ihnen möglich ist, nützen. In bezug auf Medikamente schrieb Gracián y Morales im 17. Jahrhundert: «Der Arzt brauchte gleich viel Wissenschaft zum Nichtverschreiben wie zum Verschreiben, und oft besteht die Kunst

Wir wissen aber auch, dass wir Fehler begehen - «errare humanum est» - und dass wir etwas dagegen unternehmen können und sollen. Heute sprechen wir von Patientensicherheit, Qualitätssicherung, haben Systeme zur Fehlerreduktion aus der Industrie (Du Pont) und aus der Fliegerei (Critical Incidents Reporting) in die Medizin übernommen. Patientensicherheit ist ein wichtiges Thema, viele Publikationen sind vorhanden und auch der amerikanische Präsident hat im Jahre 2000 einen 87seitigen Bericht über Patientensicherheit bekommen [1].

Medizinische Irrtümer können Schaden verursachen, es gibt dazu verschiedene Schätzungen. Die National Academy of Sciences schätzt 1999 das Ausmass für die USA auf 44000 bis 98000 Todesfälle und einen materiellen Schaden von 17 bis 29 Milliarden US-\$ [2]. Patienten mit unerwünschten Arzneimittelwirkungen kosten $32 \%$ mehr und bleiben 56\% länger im Spital [3]. Unerwünschte Arzneimittelwirkungen sind ein wesentlicher Einweisungsgrund, die Häufigkeit wird mit 7 bis $14 \%$ der Eintritte angegeben $[4,5]$. gerade in der Nichtanwendung der Mittel.»
Unerwünschte Arzneimittelwirkungen können auf medizinischen Fehlern beruhen und sind eine Form von möglicherweise vermeidbaren Schäden. Die unerwünschten Wirkungen der einzelnen Arzneimittel sind im wesentlichen bekannt. Sie sind dokumentiert in Verpackungsprospekten und in der Schweiz z. B. im «Arzneimittelkompendium».

Dagegen ist der Aufwand, um Informationen über Interaktionen zwischen verschiedenen Arzneimitteln zu bekommen, grösser. Das Risiko solcher Interaktionen steigt mit der Anzahl der Arzneimittel pro Patient [6]. Mit einem guten Teil dieser Risiken kann angemessen umgegangen werden, sofern das Risiko bekannt ist [7].

Von verschiedenen Autoren wird über die Verwendung von Computertechnologie zur Vermeidung von unerwünschten Arzneimittelwirkungen berichtet $[8,9]$ oder sie wird gefordert [10]. Informationen über Arzneimittelinteraktionen liegen in verschiedenen Datenbanken vor. Sieben davon wurden auf ihre Qualität untersucht [11].

Nach den Grundsätzen der Sicherheit (Du Pont, CIR) ist es notwendig, viele gefährliche Situationen $\mathrm{zu}$ erkennen und $\mathrm{zu}$ beheben, um wenige Schadenfälle zu vermeiden. Um viele gefährliche Situationen, wie das Risiko von Interaktionen, zu erkennen, bedarf es einfacher Massnahmen, die kostengünstig zu realisieren sind.

Wir untersuchen in dieser Arbeit die Tauglichkeit einer solchen Massnahme im klinischen Alltag. Wir erfassen die Ausbeute an Informationen über Interaktionsrisiken, die klinischen Konsequenzen, die sich aus solchen Risiken ergeben, und messen den zusätzlichen Aufwand, der betrieben werden muss.

\section{Patienten und Methoden}

Untersucht wurden mögliche Arzneimittelinteraktionen bei Patienten und Patientinnen der Rehabilitationsabteilung der Klinik Schützen in Rheinfelden. Es sind vorwiegend ältere Menschen zwischen 42 und 93 Jahre alt, die zur Hälfte postoperativ zur muskuloskelettalen Rehabilitation, zur Hälfte zur Weiterbehandlung 
nach somatischen Krankheiten oder nach Krebsoperationen hospitalisiert sind. Sie werden in der Regel aus einem Akutspital zugewiesen.

Wir haben über die Website der Apotheke zur Rose (www.aporose.ch) mit der dort zur Verfügung gestellten «Interaktionskontrolle» auf die Daten über Interaktionsrisiken zugegriffen. Auf diese Weise konnten die in der Schweiz gebräuchlichen Markennamen verwendet und unbeschränkt viele Medikamente eingegeben werden. Neben einer Kurzinformation über Interaktionsrisiken stand auch ein ausführlicher Text über dieselben zur Verfügung.

Die Interaktionsrisiken sind bewertet nach folgenden Definitionen:

\section{Schwerwiegend}

Diese Arzneimittelkombination kann für den Patienten lebensbedrohend sein, oder es können Intoxikationen oder bleibende Schädigungen für den Patienten entstehen. Die Arzneimittel sollen in der Regel nicht gleichzeitig eingenommen werden.

\section{Mittelschwer}

Die Kombination führt häufig zu therapeutischen Schwierigkeiten, doch bei sorgfältiger Überwachung des Patienten (Laborwerte, zum Beispiel Quickwert, klinische Symptome) kann die Kombination verabreicht werden.

\section{Geringfügig}

Bei geringfügigen Interaktionen können etwas verstärkte oder verminderte Wirkungen auftreten oder sie betreffen nur einen bestimmten Personenkreis (zum Beispiel Patienten mit Nierenoder Leberinsuffizienz, Langsamazetylierer).

\section{Unbedeutend}

Interaktionen, die meist keine oder nur geringe Auswirkungen haben und keine besonderen Massnahmen erfordern, erscheinen nur mit der Schnellinformation. Ausführliche Monographien sind nicht abrufbar. Dies gilt ebenso für

\section{Fremdangaben}

Diese Interaktionen sind nur in Einzelfällen beschrieben oder werden vermutet, ihre klinische Relevanz ist jedoch unklar.

Diese Arbeit wurde von August bis Oktober 2004 von einer medizinischen Praxisassistentin (MPA) routinemässig bei allen Eintritten auf der Rehabilitationsabteilung durchgeführt. Zusätzlich wurden die entsprechenden Daten aus Krankengeschichten des Jahres 2003 erhoben und zusammen mit den aktuellen Daten ausgewertet. Die
Liste der möglichen Interaktionen wurde ausgedruckt und den zuständigen Ärzten und Ärztinnen zur Verfügung gestellt. Diese legten fest, welche Massnahmen aufgrund der gefundenen Interaktionsrisiken angezeigt waren.

\section{Resultate}

Patienten und Patientinnen (Abb. 1)
$\begin{array}{ll}\text { Anzahl } & 102 \\ \text { Geschlecht } & 70 \text { Frauen, 32 Männer } \\ \text { Alter } & 42 \text { bis } 93 \text { Jahre, Mittel } 75\end{array}$

Im Mittel waren beim Eintritt 7,8 verschiedene Medikamente verordnet (Abb. 2). Bei Eintritt wurden 301 Interaktionsrisiken gefunden, davon 9 schwerwiegende (Abb. 3). Die Zahl der Interaktionsrisiken stieg mit der Zahl der Medikamente an (Abb. 4). 57 Patienten und Patientinnen mit erheblichem Risiko (1 oder mehrere Risiken der Kategorie: «schwerwiegend» und «mittelschwer») konnten identifiziert werden. Einige wiesen 5 bis 10 solcher Risiken auf (Abb. 5). Folgende Konsequenzen wurden gezogen: Am häufigsten besondere Kontrollen oder Aufmerksamkeit, seltener Wechsel oder Absetzen des Medikamentes (Abb. 6).

\section{Aufwand}

Der gesamte Aufwand (Infrastruktur und Zeit) zur Erhebung dieser Interaktionsrisiken war gering.

PC-Stationen mit Internetanschluss bestanden schon. Um auf die Interaktionskontrolle der Apotheke zur Rose zugreifen zu können braucht es ein HIN-Abonnement mit der entsprechenden ASAS-Sicherheitssoftware. Diese sind auch in vielen Arztpraxen bereits vorhanden. Weitere Infrastrukturkosten fielen nicht an.

Der Zeitaufwand der MPA betrug im ganzen 12 Arbeitsstunden, was pro Fall einen Aufwand von 7 bis 8 Minuten bedeutet und zusätzliche Personalkosten von rund Fr. 6.- pro Fall auslöste. Der Zeitaufwand der Ärztinnen und Ärzte wurde nicht berücksichtigt, da es sich dabei um normale, durch die Erhebung eher vereinfachte, klinische Tätigkeit handelte.

\section{Diskussion}

\section{Grenzen der Arbeit}

Nur was in der Datenbank erfasst ist, kann ausgewertet werden, z.B. fehlen Alkohol, Nahrungsmittel, Naturheilmittel und z. T. OTC-Medikamente. Hier bleibt eine Unsicherheit bzw. ein Lücke. 
Abbildung 1

Altersverteilung.

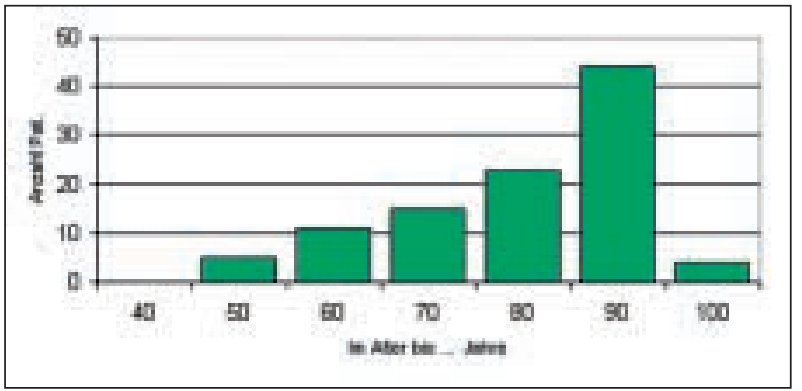

Abbildung 2

Medikamentenverordnungen pro Fall.

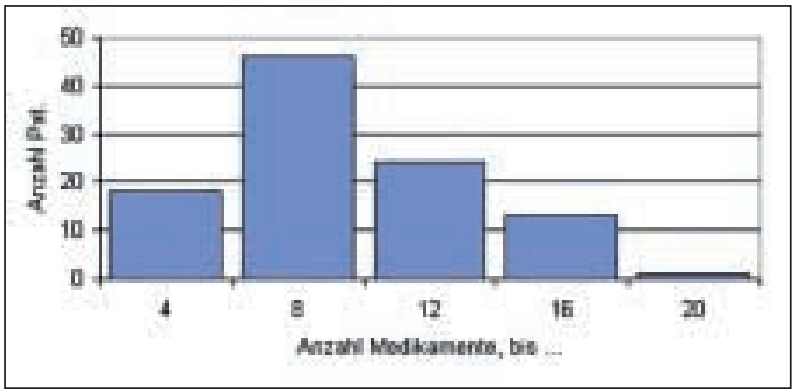

Abbildung 3

Interaktionsrisiken.

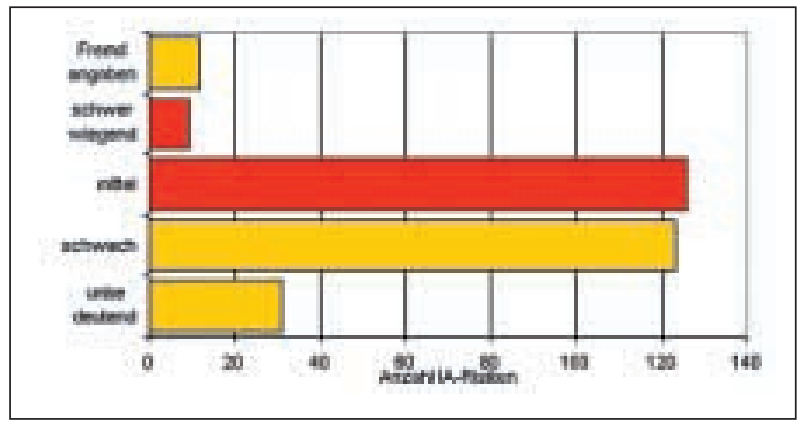

Abbildung 4

Interaktionen nach Anzahl Medikamente.

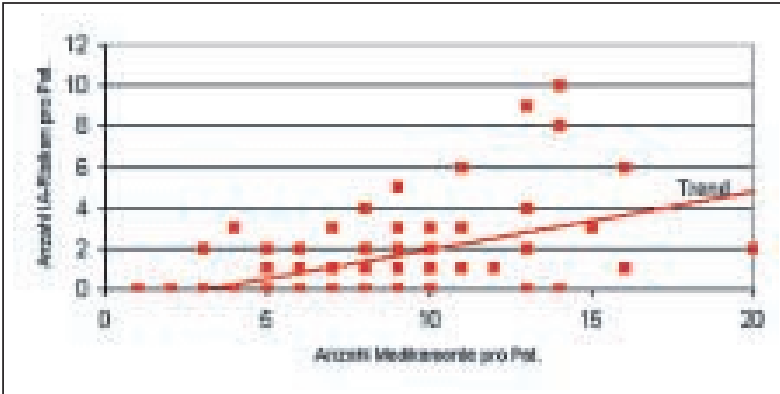

Interaktionsrisiken sind in diesem Patientenkollektiv häufig. Sie können zu einem grossen Teil mit einfachen Mitteln und wenig Aufwand auch von Nicht-Ärzten erfasst werden. Häufig ist das Interaktionsrisiko nicht $\mathrm{zu}$ vermeiden. In vielen Fällen sind Kontrollen von Laborwerten oder klinischen Befunden angezeigt, häufiger, als sie ohne Erkennen des Interaktionsrisikos durchgeführt würden. Seltener müssen Medikamente abgesetzt oder gewechselt werden.

Mit einer konsequenten Erfassung des Interaktionsrisikos kann eine grosse Anzahl von gefährlichen Situationen erkannt und angemessen reagiert werden.

Der Aufwand ist gering und der Nutzen ist beträchtlich. Im klinischen Alltag ergeben sich daraus zusätzliches Wissen und Sicherheit. Wir empfehlen, diese Kontrolle bei Patienten und Patientinnen, welche mehrere Medikamente einnehmen müssen, routinemässig durchzuführen.

\section{Danksagung}

Wir danken Frau Susanne Schmidt Schreiner für die zuverlässige und speditive Datenerfassung, Herrn lic. phil Jürg Waldmeier für die kritische Durchsicht des Manuskriptes und der Apotheke zur Rose für ihre Informationen.

\section{Literatur}

1 Report of the Quality Interagency Coordinating Task Force. To the President, February 2000. Doing what counts for patient safety: Federal actions to reduce medical errors and their Impact.

2 National Academy of Sciences, Institute of Medicine. To Err Is Human, Building a Safer Health System, erhältlich unter www.nap.edu/ books/0309068371/html/.

3 Suh DC, Woodall BS, Shin SK, Hermes-De Santis ER. Clinical and economic impact of adverse drug reactions in hospitalized patients. Ann Pharmacother 2000;34(12):1373-9.

4 Lagnaoui R, Moore N, Fach J, Longy-Boursier M, Begaud B. Adverse drug reactions in a department of systemic diseases-oriented internal medicine: prevalence, incidence, direct costs and avoidability. Eur J Clin Pharmacol 2000;56(2):181-6.

5 van Kraaij DJ, Haagsma CJ, Go IH, Gribnau FW. Drug use and adverse drug reactions in 105 elderly patients admitted to a general medical ward. Neth J Med 1994;44(5):166-73.

6 van den Bemt PM, Egberts AC, Lenderink AW, Verzijl JM, Simons KA, van der Pol WS, Leufkens HG. Risk factors for the development of adverse drug events in hospitalized patients. Pharm World Sci 2000;22(2):62-6. 
Abbildung 5

Erhebliche Risiken.

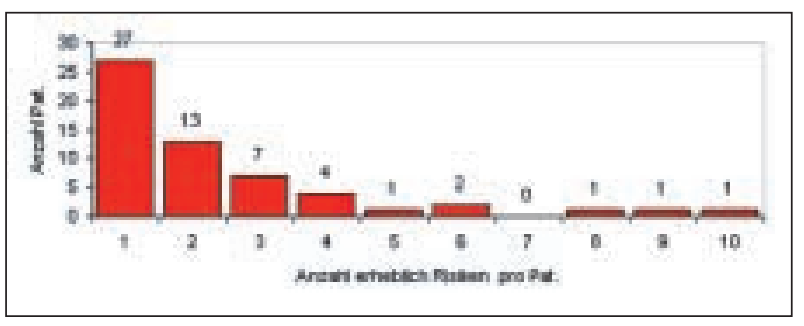

Abbildung 6

Konsequenzen.

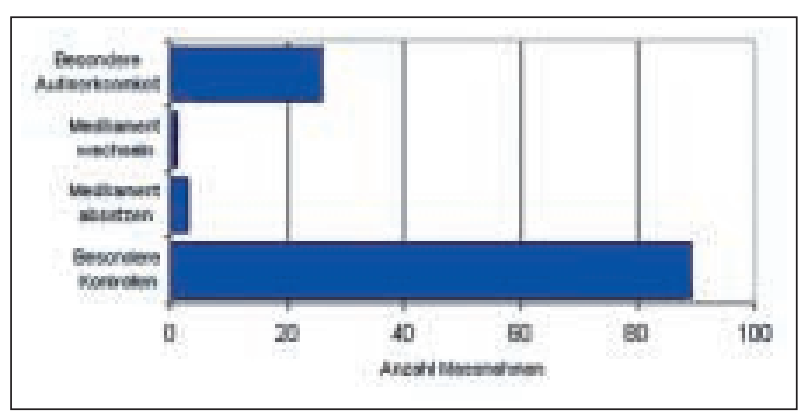

7 Bergk V, Gasse C, Rothenbacher D, Loew M, Brenner H, Haefeli WE. Drug interactions in primary care: impact of a new algorithm on risk determination. Clin Pharmacol Ther 2004;76(1): 85-96.

8 Bonetti PO, Hartmann K, Kuhn M, Reinhart WH, Wieland T. Potentielle Arzneimittelinteraktionen und Verordnungshäufigkeit von Medikamenten mit speziellem Instruktionsbedarf bei Spitalaustritt. Praxis 2000;89:182-9.

9 Jha AK, Kuperman GJ, Rittenberg E, Teich JM, Bates DW. Identifying hospital admissions due to adverse drug events using a computer-based monitor. Pharmacoepidemiol Drug Saf 2001; 10(2):113-9.

10 Lepori V, Perren A, Marone C. Unerwünschte internmedizinische Arzneimittelwirkungen bei Spitaleintritt. Schweiz Med Wochenschr 1999; 129(24):915-22.

11 Perrin Y, Biclin T, Biollaz J. Bases de données informatiques d'interactions médicamenteuses: quel choix? Praxis 2004;93:1-996. 